\title{
Estudo da atividade anti-helmíntica do extrato etanólico de Jatropha mollissima (Pohl) Baill. (Euphorbiaceae) sob Haemonchus contortus em ovinos no semiárido paraibano ${ }^{1}$
}

\author{
Ana R.C. Ribeiro ${ }^{2 *}$, Fábio D. de Andrade², Maria do C. de Medeiros² ${ }^{2}$ Alricélia da S. Camboim³ \\ Francisco A. Pereira Júnior ${ }^{2}$, Ana C.R. Athayde², Onaldo G. Rodrigues ${ }^{2}$ e Wilson W. Silva ${ }^{2}$
}

\begin{abstract}
Ribeiro A.R.C., Andrade F.D., Medeiros M.C., Camboim A.S., Pereira Jr F.A., Athayde A.C.R., Rodrigues O.G. \& Silva W.W. 2014. [Study of the anthelminthic activity of the ethanol extract of Jatropha mollissima (Pohl) Baill. (Euphorbiaceae) on Haemonchus contortus in sheep of the Paraiba semiarid, Brazil.] Estudo da atividade anti-helmíntica do extrato etanólico de Jatropha mollissima (Pohl) Baill. (Euphorbiaceae) sob Haemonchus contortus em ovinos no semiárido paraibano. Pesquisa Veterinária Brasileira 34(11):1051-1055. Programa de Pós-Graduação em Zootecnia, Universidade Federal de Campina Grande, Rodovia Patos-Teixeira, Km Zero, Jatobá, Patos PB 58700-970, Brazil. E-mail: a.raquel.ribeiro@hotmail.com

This study aimed to evaluate the anthelmintic effect of Jatropha mollissima through in vitro and in vivo experiments. Initially we investigated the concentration of extract with bioactive effect, through the toxicity evolution test of the ethanol extract of J. mollissima on the microcrustacean Artemia salina, obtaining CL50 concentration of $660.80 \mu \mathrm{g} / \mathrm{ml}$, which was tested in fecal cultures containing infective larvae of Haemonchus contortus and in animals for the verification of OPG reduction. For in vivo test, the extract was dissolved in water to obtain concentrations of $660.80 \mu \mathrm{g} / \mathrm{ml}$ and $1321.6 \mu \mathrm{g} / \mathrm{ml}$. Feces were collected weekly and blood was collected every fifteen days. As a result of in vitro test, the ethanol extract of the stem of J. mollissima proved toxic on A. salina, with CL50 less than 1000 $\mu \mathrm{g} /$ $\mathrm{ml}$ and inhibited the eggs hatching and the development of larvae of $H$. contortus, presenting an efficiency of $70.77 \%$. In vivo test revealed that the extract is also effective in sheep, with a significant reduction in the count of OPG after 28 days of experiment, 47 and $44 \%$ of reduction in the groups treated with the extract, $7.5 \%$ in the untreated group of animals and $40.6 \%$ with ivermectin. Even parasitized, the animals remained clinically healthy and without anemia. The ethanol extract of the stem of Jatropha mollissima may represent an alternative to the control of sheep worms, because it slows the parasitic resistance.
\end{abstract}

INDEX TERMS: Medicinal plants, Jatropha mollissima, Euphorbiaceae, Haemonchus contortus, sheep, secondary metabolites, gastrointestinal nematodes.

RESUMO.- Objetivou-se com o presente trabalho, avaliar o efeito anti-helmíntico de Jatropha mollissima por meio de experimentos in vitro e in vivo. Inicialmente foi inves-

\footnotetext{
${ }^{1}$ Recebido em 25 de março de 2014.

Aceito para publicação em 9 de agosto de 2014.

${ }^{2}$ Programa de Pós-Graduação em Zootecnia, Universidade Federal de Campina Grande (UFCG), Rodovia Patos-Teixeira Km Zero, Jatobá, Patos, PB 58700-970, Brasil. E-mails: medvetfabio@hotmail.com; ducamedeiros @hotmail.com; avelar.junior@yahoo.com.br; onaldo@cstr.ufcg.edu.br; wouflan@hotmail.com; athayde@cstr.ufcg.br; *Autor para correspondência: a.raquel.ribeiro@hotmail.com

${ }^{3}$ Laboratório de Patologia Clínica, Hospital Veterinário, UFCG, Rodovia Patos-Teixeira Km Zero, Jatobá, Patos, PB. E-mail: alriceliamv@hotmail.com
}

tigada a concentração de extrato com efeito bioativo, pelo teste de evolução da toxicidade do extrato etanólico de $J$. mollissima sobre o microcrustáceo Artemia salina, obtendo uma CL50 de $660,80 \mu \mathrm{g} / \mathrm{ml}$, que foi testada em coproculturas contendo larvas infectantes de Haemonchus contortus e em animais para a verificação da redução do OPG. Para o teste in vivo o extrato foi dissolvido em água para se obter as concentrações $660,80 \mu \mathrm{g} / \mathrm{ml}$ e $1321,6 \mu \mathrm{g} / \mathrm{ml}$, foram coletadas fezes semanalmente e sangue quinzenalmente. Como resultados dos testes in vitro, o extrato etanólico do caule de Jatropha mollissima mostrou-se tóxico sobre A. salina, com CL50 abaixo de $1000 \mu \mathrm{g} / \mathrm{ml}$ e inibiu a eclosão de ovos e o desenvolvimento de larvas de H. con- 
tortus, apresentando uma eficiência de $70,77 \%$. 0 teste in vivo revelou que o extrato é também eficaz em ovinos, com redução significativa na contagem de OPG após 28 dias de experimento, 47 e $44 \%$ de redução nos grupos tratados com o extrato, $7,5 \%$ no grupo de animais não tratados e $40,6 \%$ com a ivermectina. Mesmo parasitados, os animais permaneceram clinicamente saudáveis e sem anemia. 0 extrato etanólico do caule de Jatropha mollissima pode representar uma alternativa ao controle da verminose ovina, pois retarda a resistência parasitária.

TERMOS DE INDEXAÇÃO: Plantas medicinais, Jatropha mollissi$m a$, Euphorbiaceae, Haemonchus contortus, ovinos, metabólitos secundários, nematódeos gastrintestinais.

\section{INTRODUÇÃO}

A criação de pequenos ruminantes constitui importante fonte de renda para a região nordeste (Nascimento et al. 2013). No entanto, problemas nutricionais, de manejo e sanitários limitam a produção, gerando perdas econômicas e diminuição da rentabilidade dos rebanhos (Lima et al. 2010).

A maior parcela de prejuízos para o setor produtivo deve-se às helmintoses gastrintestinais (Vieira et al. 2014). Em ovinos, o parasita mais frequente é Haemonchus contortus, causando perda de peso, anemia e até mesmo a morte de animais mais susceptíveis (Fortes et al. 2013). 0 controle geralmente é feito empregando-se anti-helmínticos sintéticos, os quais administrados de maneira inadequada podem selecionar linhagens de parasitas resistentes (Santos et al. 2012), além poluir o meio ambiente e aumentar significativamente os custos de produção (Kunsa \& Abebe 2009).

Diante os aspectos negativos causados por moléculas de ação anti-helmíntica, estudos estão sendo desenvolvidos como alternativa de controle parasitário (Oliveira et al. 2011), nesta direção pesquisas em etnoveterinária tem mostrado o uso de inúmeras plantas medicinais na prevenção e controle de doenças dos animais e com comprovada atividade anti-helmíntica (Barrabí-Puerta \& Arece-García 2013). Outro aspecto importante no uso de plantas é que além de possuírem compostos bioativos com propriedades medicinais, apresentam baixo custo (Sousa et al. 2013).

Plantas tidas como medicinais tem sido investigadas quanto às suas propriedades (Hassum et al. 2013), dentre elas a espécie Jatropha mollissima (Pohl) Baill., popularmente conhecida como Pinhão-bravo, pertence à família Euphorbiaceae e é endêmica da Caatinga (Queiroz et al. 2013), diversos trabalhos tem demonstrado que esta espécie apresenta efeito antibacteriano (Rocha \& Dantas 2009), hipotensor e estimulante dos músculos lisos do intestino e do útero (Leal \& Agra 2005) e antioxidante (Melo et al. 2010).

Nesse contexto, o presente trabalho objetivou avaliar o efeito anti-helmíntico de Jatropha mollissima através de experimento in vitro e in vivo sobre o nematódeo Haemonchus contortus e seu efeito sobre o hematócrito de ovinos experimentalmente infectados em região de caatinga.

\section{MATERIAL E MÉTODOS}

\section{Coleta do material botânico}

Os procedimentos de coleta e herborização do material vegetal foram realizados baseando-se nas metodologias de Cartaxo et al. (2010). A planta foi coletada na microrregião dos Inhamuns (Tauá-Ceará), $\left(040^{\circ} 18^{\prime} 05,4^{\prime \prime} \mathrm{W}\right.$; 0601'03,6” S), identificada e depositada no Herbário Carirense Dárdano de Andrade-Lima da Universidade Regional do Cariri-URCA, com exsicata nํ6675.

\section{Obtenção do extrato orgânico}

Para confecção do extrato etanólico, utilizou-se o caule de J. mollissima, sendo empregada a metodologia descrita por Matos (2000). Utilizou-se etanol 96\% como solvente orgânico e após 96h de extração a frio, foram realizadas filtrações simples e os extratos mantidos à temperatura ambiente $\left(30 \pm 2^{\circ} \mathrm{C}\right)$ para evaporação total do solvente e obtenção do extrato líquido, em seguida procedeu-se a concentração do extrato em evaporador rotativo a temperatura de $45^{\circ} \mathrm{C}$.

\section{Experimento in vitro}

Toxicidade do extrato botânico de Jatropha mollissima sobre Artemia salina. Para analise da evolução da toxicidade do extrato do caule da J. mollissima, foi utilizada a metodologia descrita por Araújo et al. (2010). 0 extrato bruto foi diluído em solução de dimetil sulfóxido (DMSO) a 1\%, nas concentrações 100, 500 e $1000 \mu \mathrm{g} / \mathrm{mL}$. Utilizaram-se três grupos controle: GC1 (Grupo Controle 1: DMSO a 1\%); GC2 (Grupo Controle 2: Solução salina isotônica); GC3 (Grupo controle 3: NaClO a 1\%).

Os valores obtidos com a média do número de náuplios mortos foram submetidos à análise estatística, em que a concentração letal foi estimada em 50\% (CL50) das larvas pelo método de Análise de Probit, de acordo com o teste de Spearman-Karber com intervalos de confiança de 95\%, usando o software TRIMMED (versão 1,5).

Ação biológica do extrato etanólico de Jatropha mollissima sobre ovos e larvas de Haemonchus contortus. Para testar a ação ovicida e larvivida in vivo, foram realizadas coproculturas utilizando a metodologia adaptada de Robert O'Sullivan (1950). Foi adicionado $2,5 \mathrm{ml}$ de extrato etanólico de J. mollissima na concentração de 660,80 $\mu \mathrm{g} / \mathrm{ml}$ à culturas fecais mono específicas de Haemonchus contortus, contendo aproximadamente 6.500 ovos. Como grupo controle (GC), substituiu-se o extrato por água destilada; todos os tratamentos foram realizados em triplicatas. Após sete dias, em temperatura ambiente, procedeu-se a recuperação e contagem das larvas de terceiro estágio (L3) em microscópio óptico.

Para avaliação da eficiência do extrato sobre ovos e larvas nos diversos tratamentos, foi utilizada a fórmula adaptada descrita por Camurça-Vasconcelos et al. (2007):

Onde:

$$
\begin{aligned}
& \text { ET: L3 inicial - L3 do grupo tratado } \\
& \text { L3 inicial }
\end{aligned}
$$

L3 inicial corresponde à estimativa do número de larvas em cada coprocultura

L3 do grupo tratado corresponde à quantidade de larvas recuperadas após 8 dias de incubação com os diferentes tratamentos.

\section{Experimento in vivo}

Local da pesquisa. 0 experimento foi conduzido no Núcleo de Pesquisa para o Tropico Semiárido - NUPEÁRIDO, do Centro de Saúde e Tecnologia Rural da UFCG - Patos, localizado no semiárido paraibano, com clima predominante seco e temperatura média anual de $30,6^{\circ} \mathrm{C}\left(28,7^{\circ} \mathrm{C}\right.$ e máxima de $\left.32,5^{\circ} \mathrm{C}\right)$. Os ensaios laboratoriais foram realizados no Laboratório Multiusuário de Pesquisas Ambientais (LAMPA), Universidade Federal de Campina Grande, PB. 
Período de adaptação. Trinta dias antes do inicio do experimento, os animais foram estabulados em baias individuais, com piso ripado suspenso, água e alimento ad libitum, vermifugados com ivermectina $1 \%$ injetável e closantel $10 \%$ via oral até a negativação do OPG. Foram administradas, em três dias consecutivos, por via oral, três mil larvas infectantes de Haemonchus contortus. Vinte um dias após a administração da última dosagem de larvas infectantes, foram realizadas contagens de ovos e os animais com OPG acima de 1000 foram selecionados para o experimento.

Delineamento experimental. Foram utilizados 20 ovinos sem padrão de raça definido (SPRD), com idade média de 12 meses, distribuídos aleatoriamente em quatro grupos e cinco repetições por tratamento (Quadro 1).

Analise parasitológica. Amostras de fezes foram coletadas diretamente da ampola retal nos dias $0,7,14,21$ e 28 e encaminhadas ao LAMPA, para serem processadas no setor do Laboratório de Doenças Parasitárias dos Animais Domésticos (LDPAD), da Universidade Federal de Campina Grande (UFCG), Centro de Saúde e Tecnologia Rural (CSTR), para contagem de ovos (Ueno \& Gonçalves 1998) e coprocultura (Roberts \& O'Sulivan 1950).

Hematócrito. Amostras de sangue foram coletadas diretamente da veia jugular de cada animal, nos dias 0,14 e 28 do período experimental e acondicionadas em tubos estéreis a vácuo contendo EDTA (etilenodiamino-tetracético-di-sódico) como anticoagulante. Após coletadas, as amostras foram encaminhadas ao Laboratório de Patologia Clinica Veterinária do Hospital Veterinário da Universidade Federal de Campina Grande, para verificação do Hematócrito (Ht) (Jain 1993).

Analise estatística. Os valores de OPG foram analisados após a transformação logarítmica em log $(\mathrm{x}+1)$ e submetidos à análise de variância (ANOVA) de uma via e teste de Tukey a 5\% de probabilidade. As análises foram realizadas utilizando o software BIOESTAT 5.0 (Ayres et al. 2007). Para determinação da eficiência do extrato etanólico da J. mollissima, foi utilizado o teste de redução de ovos por grama de fezes (RCOF), descrita por Coles et al. (1992):

Onde:

$$
\mathrm{RCOF}=[1-(\mathrm{OPGi} / \mathrm{OPGf})] \times 100
$$

OPGf = média do número de ovos por grama de fezes no final do tratamento;

OPGi = média do número de ovos por grama de fezes no início do tratamento.
As análises hematológicas foram avaliadas por ANOVA seguido do teste Tukey. Os resultados foram processados no programa estatístico SAS (versão 9.1) e o nível de significância foi 5\%.

\section{RESULTADOS E DISCUSSÃO}

\section{Experimento in vitro}

0 teste de toxicidade revelou que o extrato de Jatropha mollissima é tóxico mesmo em baixas concentrações, apresentando CL50 igual a 660,80 $\mathrm{gg} / \mathrm{ml}$ (Quadro 2), resultado que comprova a ação bioativa desse extrato, de acordo com a classificação descrita por Mayer (1982), que considera tóxicos extratos que apresentam CL50 inferior a $1000 \mu \mathrm{g} /$ $\mathrm{ml}$. Rajeh et al. (2012) verificaram o efeito tóxico do extrato do caule de Euphorbia hirta, também do gênero Euphorbiaceae, nas concentrações de $0,07-100 \mathrm{mg} / \mathrm{ml}$, obtendo como resultado, a CL50 1,589mg/ml. Quando comparados com resultados obtidos no presente trabalho, verifica-se a $J$. mollissima foi mais eficiente, provavelmente porque os valores dos princípios bioativos são maiores.

A concentração letal para 50\% (CL50) é um parâmetro utilizado em testes para a detecção de substâncias bioativas e os resultados de seu estudo relacionam-se a efeito anti-helmíntico (Amarante et al. 2011). Dessa forma, no presente estudo utilizou como parâmetro inicial a CL50 dos náuplios de Artemia salina, e somente após os resultados investigou-se a sua ação biológica sobre o nematódeo Haemonchus contortus de ovinos.

$\mathrm{Na}$ análise das culturas fecais, observa-se que nas coproculturas em que foi adicionado o extrato etanólico de J. mollissima, houve redução estatisticamente significativa $(p>0,05)$ na eclodibilidade, quando comparada com a coprocultura do grupo controle (Quadro 3). Quanto à recuperação das larvas de terceiro estágio, as coproculturas que receberam o extrato tiveram uma eficiência de $70,77 \%$ (1900) e no grupo controle a eficiência foi de apenas $10,4 \%$ (5.824), sugerindo que o caule do pinhão-bravo pode ter efeito anti-helmíntico em animais.

\section{Quadro 1. Delineamento do experimento in vivo com ovinos SRD submetidos a tratamentos com Jatropha mollissima no semiárido paraibano}

\begin{tabular}{ccccccc}
\hline \multicolumn{3}{c}{ Grupo Animais Tratamento } & Concentração & Dosagem & \multicolumn{2}{c}{ Aplicação } \\
\cline { 5 - 6 } & & & & Via & Dia \\
\hline G1 & 5 & Ivermectina & $1 \%$ & $1 \mathrm{ml} / \mathrm{Kg} / \mathrm{PV}$ & Parenteral & Zero \\
G2 & 5 & Água & 0 & $10 \mathrm{ml}$ & Oral & $0,7,14,21$ e 28 \\
G3 & 5 & Extrato & $660,80 \mu \mathrm{g} / \mathrm{ml}$ & $10 \mathrm{ml}$ & Oral & $0,7,14,21$ e 28 \\
G4 & 5 & Extrato & $1321,6 \mu \mathrm{g} / \mathrm{ml}$ & $10 \mathrm{ml}$ & Oral & $0,7,14,21$ e 28
\end{tabular}

Quadro 2. Resultados do teste de toxicidade para o extrato do caule de Jatropha mollissima sobre Artemia salina

\begin{tabular}{ccccccc}
\hline $\begin{array}{c}\text { Concentração } \\
\text { do Extrato }(\mu \mathrm{g} / \mathrm{mL})\end{array}$ & \multicolumn{3}{c}{$\begin{array}{c}\text { Número de Óbitos } \\
\text { de A. salina }\end{array}$} & CL50 & $\begin{array}{c}\text { Intervalo de } \\
\text { Confiança }\end{array}$ \\
\cline { 2 - 5 } & GTECJM & GC1 & GC2 & GC3 & & \\
\hline 100 & 0 & 1 & 3 & 19 & 660,80 & $598,57-729,51$ \\
500 & 2 & 4 & 3 & 19 & & \\
1000 & 30 & 7 & 3 & 19 & & \\
\hline
\end{tabular}

GTECJM = Grupo Tratado com Extrato do Caule de Jatropha mollissima; GC1 = Grupo Controle 1: DMSO a 1\%; GC2 = Solução salina isotônica; GC3 = Grupo controle 3: NaClO a 1\%; CL50 = Concentração letal para $50 \%$ dos náuplios de Artemia salina.
Quadro 3. Eficiência do extrato etanólico do caule de Jatropha mollissima sobre Haemonchus contortus de ovinos

\begin{tabular}{lcccc}
\hline Tratamento & $\begin{array}{c}\text { Concentração } \\
(\mu \mathrm{g} / \mathrm{ml})\end{array}$ & $\begin{array}{c}\text { Média de } \\
\text { L3 }\end{array}$ & $\begin{array}{c}\text { Eclodibilidade } \\
(\%)\end{array}$ & $\begin{array}{c}\text { Eficiência } \\
(\%)\end{array}$ \\
\hline Extrato & 660,80 & $1900+430$ & 29,23 & 70,77 \\
Controle & Água destilada & $5824+410$ & 89,6 & 10,4
\end{tabular}

Monteiro et al. (2011), obtiveram resultados semelhantes, ao testar o efeito in vitro do extrato etanólico de Jatropha curcas, e observaram redução de 99,8\% na eclodibilidade com o extrato na concentração $50 \mathrm{mg} / \mathrm{mL}$, enquanto no presente estudo foi de $89,6 \%$ a uma concentração infe- 
Quadro 4. Média aritmética re-transformada [(log $(x+1)]$, desvio padrão e percentual de redução do OPG de ovinos artificialmente infectados com Haemonchus contortus, submetidos a diversos tratamentos com extrato etanólico de Jatropha mollissima

\begin{tabular}{ccccccc}
\hline Tratamentos & OPG Dia zero & OPG Dia 07 & OPG Dia 14 & OPG Dia 21 & OPG Dia 28 & RCOF (\%) \\
\hline G1 & $1500^{\mathrm{a}}+320$ & $1433^{\mathrm{a}}+350$ & $1180^{\mathrm{a}}+420$ & $1280^{\mathrm{a}}+250$ & $890^{\mathrm{b}}+410$ & 40,61 \\
G2 & $1460^{\mathrm{a}}+250$ & $1550^{\mathrm{a}}+300$ & $1900 \mathrm{a}+300$ & $1620^{\mathrm{a}}+300$ & $1350^{\mathrm{a}}+200$ & 7,52 \\
G3 & $1400^{\mathrm{a}}+300$ & $920^{\mathrm{b}}+240$ & $860^{\mathrm{b}}+200$ & $640^{\mathrm{b}}+360$ & $740^{\mathrm{b}}+300$ & 47,11 \\
G4 & $1600^{\mathrm{a}}+280$ & $1200^{\mathrm{a}}+300$ & $1160^{\mathrm{b}}+300$ & $1080 \mathrm{~b}+260$ & $900^{\mathrm{b}}+200$ & 43,71
\end{tabular}

$\overline{\text { Valores seguidos }}$ por letras diferentes em linhas diferem estatisticamente $(p>0,05)$ pelo Teste Tukey para amostras independentes, valores seguidos de número diferentes nas colunas diferem estatisticamente ( $\mathrm{p}>0,05)$. G1 = Controle positivo, Ivermectina 1\%; G2 = Controle negativo, água; G3 = Pinhão 660,80 $\mu \mathrm{g} / \mathrm{ml} ; \mathrm{G} 4$ = Pinhão 1321,6 $\mu \mathrm{g} / \mathrm{ml}$.

rior $(660,80 \mu \mathrm{g} / \mathrm{ml})$. 0 maior percentual observado no trabalho citado, pode ter ocorrido devido ao fato de ter sido utilizada uma maior concentração do extrato ou ainda devido à variação na intensidade de metabólitos secundários presentes naquela planta (Sabandar et al. 2013).

\section{Eficácia do experimento in vivo}

$\mathrm{Na}$ avaliação do efeito do extrato etanólico de Jatropha mollissima sobre os percentuais de redução da carga parasitária in vivo, observou-se redução de 47,1 e 43,7\% na contagem de ovos por grama de fezes (OPG) dos grupos tratados com o extrato nas concentrações $660,80 \mu \mathrm{g} / \mathrm{ml}$ e $1321,6 \mu \mathrm{g} / \mathrm{ml}$, respectivamente, enquanto no controle com ivermectina $1 \%$, a redução foi de 40,6\% (Quadro 4). 0 grupo de animais tratados com a concentração de $660,80 \mu \mathrm{g} / \mathrm{ml}$ apresentou redução da carga parasitária dos animais (OPG) desde a primeira semana de tratamento, no entanto, ao final do experimento, o percentual de redução(RCOF) das duas concentrações de extrato utilizadas $(660,80 \mu \mathrm{g} / \mathrm{ml}$ e $1321,6 \mu \mathrm{g} / \mathrm{ml})$ foram estatisticamente iguais e superiores ao controle negativo (água).

Nos grupos dos animais tratados com o extrato e ivermectina (Quadro 1), observou-se que os valores de OPG decresceram significativamente $(p>0,05)$ ao longo do experimento, o que não foi observado no controle negativo. 0 baixo percentual observado no grupo de animais tratos com ivermectina pode ter ocorrido devido à resistência parasitária (Almeida et al. 2010), onde o fármaco que é constantemente empregado, seleciona linhagens de helmintos resistentes. Segundo Barrère et al. (2013), a resistência representa um grande problema na criação de pequenos ruminantes em todo o mundo. No Nordeste brasileiro, Santos et al. (2014) também observaram este fenômeno em $H$. contortus. Nesse sentido, pesquisas têm buscado alternativas para o controle parasitário (Ribeiro et al. 2013).

Na literatura consultada, não há relatos de pesquisas semelhantes às do presente trabalho, com a espécie $J$. mollissima. Porém, estudos apontam a eficácia anti-helmíntica de plantas da mesma família. No Brasil, Carvalho et al. (2012), constataram a eficácia in vitro de Hura crepitans na inibição do desenvolvimento larval de Haemonchus contortus em ovinos e Lone et al. (2012), in vivo com Euphorbia helioscopia.

Quanto à avaliação do hematócrito (Ht) (Quadro 5), observou-se que os valores encontrados estão dentro dos parâmetros considerados normais para a espécie, $27-45 \%$ (Byers \& Kramer 2010), nos grupos submetidos aos tratamentos com J. mollissima, ivermectina e controle negativo. Costa et al.
Quadro 5. Valores percentuais médios do Hematócrito (Ht) de ovinos artificialmente infectados com Haemonchus contortus nos diversos tratamentos

\begin{tabular}{ccccc}
\hline Dia & G1 & G2 & G3 & G4 \\
\hline 0 & $33,20 \pm 2,39^{\mathrm{a}}$ & $34,60 \pm 1,95^{\mathrm{a}}$ & $35,80 \pm 2,39^{\mathrm{a}}$ & $34,40 \pm 3.58^{\mathrm{a}}$ \\
14 & $34,80 \pm 2,59^{\mathrm{a}}$ & $35,40 \pm 2,30^{\mathrm{a}}$ & $32,00 \pm 2,00^{\text {a }}$ & $30,60 \pm 4.16^{\mathrm{a}}$ \\
28 & $34,20 \pm 1,92^{\mathrm{a}}$ & $34,20 \pm 1,30^{\mathrm{a}}$ & $32,60 \pm 3,049^{\mathrm{a}}$ & $29,80 \pm 5.26^{\mathrm{a}}$
\end{tabular}

Valores seguidos por letras diferentes em linhas diferem estatisticamente $(\mathrm{p}>0,05)$ pelo Teste de Tukey para amostras independentes. G1 = Controle positivo, Ivermectina 1\%; G2 = Controle negativo, água; G3 = Pi-

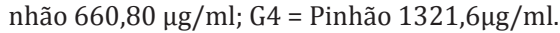

(2011), avaliando ovinos tratados com closantel e ivermectina, também não observaram variação significativa no hematócrito após a aplicação dos tratamentos. Kaplan (2004), afirma que a infecção por $H$. contortus causa anemia aos animais devido à ação espoliativa do parasita adulto, ingerindo até $250 \mathrm{~mL}$ de sangue por dia (Urquhart, 1996). Os animais do presente estudo, mesmo parasitados, não apresentaram anemia, segundo Molento et al. (2004), animais com OPG acima de 1000 não apresentam sinais clínicos de anemia. Isto pode ter ocorrido provavelmente devido capacidade de recuperação do VG dos animais (Madureira et al. 2013). De acordo com Delfino et al. (2012) no início do parasitismo, órgãos eritropoiéticos como a medula óssea, dos ossos longos, são ativados e os valores sanguíneos retornam à sua normalidade.

\section{CONCLUSÕES}

O extrato etanólico do caule de Jatropha mollissima na concentração de $660 \mu \mathrm{g} / \mathrm{mL}$ é tóxico para náuplios de $A r$ temia salina.

As concentrações $660 \mu \mathrm{g} / \mathrm{mL}$ e $1321,6 \mu \mathrm{g} / \mathrm{ml}$ do extrato etanólico do caule de J. mollissima são eficazes sobre o nematódeo Haemonchus contortus de ovinos.

A planta estudada demonstra ter potencial fitoterápico para fins de controle de nematódeos gastrintestinais em ovinos.

\section{REFERÊNCIAS}

Almeida F.A., Garcia K.C.O.D., Torgerson P.R. \& Amarante A.F.T. 2010. Multiple resistance to anthelmintics by Haemonchus contortus and Trichostrongylus colubriformis in sheep in Brazil. Parasitol. Int. 59:622-625.

Amarante C.B., Müller A.H., Póvoa M.M. \& Dolabela M.F. 2011. Estudo fitoquímico biomonitorado pelos ensaios de toxicidade frente à Artemia salina e de atividade antiplasmódica do caule de aninga (Montrichardia linifera). Acta Amazonica 41:431-434.

Araújo M.G.F., Cunha W.R. \& Veneziani R.C.S. 2010. Estudo fitoquímico pre- 
liminar e bioensaio toxicológico frente a larvas de Artemia salina Leach. de extrato obtido de frutos de Solanum lycocarpum A. St.-Hill. (Solanaceae). Revta Ciênc. Farm. Básica Apl. 31:205-209.

Ayres M., Ayres Júnior M., Ayres D.L. \& Santos A.A. 2007. BIOESTAT: aplicações estatísticas nas áreas das ciências bio-médicas. Ong Mamiraua. Belém, PA.

Barrabí-Puerta M. \& Arece-García J. 2013. Actividad antihelmíntica in vitro de extracto acuoso de hojas y semillas de Neem (Azadirachta indica A. Juss.). I. Inhibición de la eclosión de huevos y del desarrollo larvário. Revta Salud Anim. 35:103-108.

Barrère V., Keller K., Samson-Himmelstjerna G.V. \& Prichard R.K. 2013. Efficiency of a genetic test to detect benzimidazole resistant Haemonchus contortus nematodes in sheep farms in Quebec, Canada. Parasitol. Int. 62:464-470.

Byers S.R. \& Kramer J.W. 2010. Normal hematology of sheep and goats, p.836-842. In: Weiss D.J. \& Wardrop K.J. (Eds), Schalm's Veterinary Hematology. $6^{\text {th }}$ ed. Wiley-Blackwell, Ames, Iowa.

Camurça-Vasconcelos A.L.F., Bevilaqua C.M.L., Morais S.M., Maciel M.V., Costa C.T.C., Macedo I.T.F., Oliveira L.M.B., Braga R.R., Silva R.A. \& Vieira L.S. 2007. Anthelmintic activity of Croton zehntneri and Lippia sidoides essential oils. Vet. Parasitol. 148:288-294.

Cartaxo S.L., Souza M.M.A. \& Albuquerque U.P. 2010. Medicinal plants with bioprospecting potential used in semi-arid Northeastern Brazil. J. Ethnopharmacol. 131:326-342.

Carvalho C.O., Chagas A.C.S., Cotinguiba F., Furlan M., Brito L.G.l., Chaves F.C.M., Stephan M.P., Bizzo H.R. \& Amarante A.F.T. 2012. The anthelmintic effect of plant extracts on Haemonchus contortus and Strongyloides venezuelensis. Vet. Parasitol. 183:260-268.

Coles G.C., Bauer C., Borgsteede F.H.M., Geerts S., Klei T.R., Taylor M.A. \& Waller P.J. 1992. World Association for the Advancement of Veterinary Parasitology (WAAVP) methods for the detection of anthelmintic resistance in nematodes of veterinary importance. Vet. Parasitol. 44:35-44.

Costa K.M.F.M., Ahid S.M.M., Vieira L.S., Vale A.M. \& Soto-Blanco B. 2011. Efeitos do tratamento com closantel e ivermectina na carga parasitária, no perfil hematológico e bioquímico sérico e no grau Famacha de ovinos infectados com nematódeos. Pesq. Vet. Bras. 31:1075-1082.

Delfino L.J.B., Souza B.B., DA R.M.M. \& Silva W.W. 2012. Efeito do estresse calórico sobre o eritrograma de ruminantes. Revta ACSA 8:1-7.

Fortes F.S., Kloster F.S., Schafer A.S., Bier D., Buzatti A., Yoshitani U.Y. \& Molento M.B. 2013. Evaluation of resistance in a selected field strain of Haemonchus contortus to ivermectin and moxidectin using the Larval Migration on Agar Test. Pesq. Vet. Bras. 33:183-187.

Hassum I.C., Venturi C.R., Gosmann G. \& Deiro A.M.G. 2013. Ação dos extratos de quatro plantas sobre larvas infectantes de nematódeos gastrintestinais de ovinos. Revta Cubana Plant. Med. 18:278-287.

Jain N.C. 1993. Essentials of Veterinary Hematology. Lea and Febiger, Philadelphia. $417 \mathrm{p}$.

Kaplan R.M. 2004. Drug resistance in nematodes of veterinary importance: a status report. Trends Parasitol. 20:477-481.

Kunsa B. \& Abebe G. 2009. Multi antielmintic resistance of goat farm in Hawassa (South Ethiopia). Trop. Anim. Health Prod. 41:655-662.

Leal C.K.A. \& Agra M.F. 2005. Estudo farmacobotânico comparativo das folhas de Jatropha mollissima (Pohl) Baill. e Jatropha ribifolia (Pohl) Baill. (Euphorbiaceae). Acta Farm. Bonaer. 24:5-13.

Lima W.C., Athayde A.C.R., Medeiros G.R., Lima D.A.S.D., Borburema J.B., Santos E.M., Vilela V.L.R. \& Azevedo S.S. 2010. Nematoides resistentes a alguns anti-helmínticos em rebanhos caprinos no Cariri Paraibano. Pesq. Vet. Bras. 30:1003-1009.

Lone B.A., Chishti M.Z., Bhat F.A., Tak H. \& Bandha S.A. 2012. In vitro and in vivo anthelmintic activity of Euphorbia helioscopia L. Vet. Parasitol. 189:317-321.

Madureira K.M., Gomes V., Barcelos B., Zani B.H., Shecaira C.L., Baccili C.C. \& Benesi F.J. 2013. Parâmetros hematológicos e bioquímicos de ovinos da raça Dorper. Semina, Ciênc. Agrárias 34:811-816.
Matos F.J.A. 2000. Introdução a Fitoquímica Experimental. $2^{\mathrm{a}}$ ed. Universidade Federal do Ceará, Fortaleza. 141p.

Melo J.G., Araújo T.A.S., Castro V.T.N.A., Cabral D.L.V., Rodrigues M.D., Nascimento S.C., Amorim E.L.C. \& Albuquerque U.P. 2010. Antiproliferative activity, antioxidant capacity and tannin content in plants of semi-arid northeastern Brazil. Molecules 15:8534-8542.

Meyer B.N., Ferrigni N.R., Putnan J.E., Jacobsen L.B., Nichols D.E. \& McLaughlin J. 1982. Brine shrimp: a convenient general bioassay for active plant constituents. J. Med. Plant Res. 45:31-34.

Molento M.B. 2004. Resistência de helmintos em ovinos e caprinos. Revta Bras. Parasitol. Vet. 13:82-87.

Monteiro M.V.B., Bevilaqua C.M.L., Morais S.M., Machado L.K.A., Camurça-Vasconcelos A.L.F., Campello C.C., Ribeiro W.L.C. \& Mesquita M.A. 2011. Anthelmintic activity of Jatropha curcas L. seeds on Haemonchus contortus. Vet. Parasitol. 182:259-263.

Nascimento M.C.O., Souza B.B., Silva F.V. \& Melo T.S. 2013. Armazenamento de forragem para caprinos e ovinos no semiárido do nordeste. Agropec. Cient. Semiárido 9:20-27.

Oliveira L.M.B., Bevilaqua C.M.L., Morais S.M., Camurça-Vasconcelos A.L.F. \& Macedo I.T.F. 2011. Plantas taniníferas e o controle de nematóides gastrintestinais de pequenos ruminantes. Ciência Rural 41: 1967-1974.

Queiroz M.F., Fernandes P.D., Dantas Neto J., Arriel N.H.C., Francisco J.L., Marinho F.J.L. \& Leite S.F. 2013. Crescimento e fenologia de espécies de Jatropha durante a estação chuvosa. Revta Bras. Eng. Agríc. Ambient. 17:405-411.

Rajeh M.A., Kwan Y.P., Zakaria Z., Latha L.Y., Jothy S.L. \& Sasidharan S. 2012. Acute toxicity impacts of Euphorbia hirta L. extract on behavior, organs body weight index and histopathology of organs of the mice and Artemia salina. Pharmacogn. Res. 4:170-177.

Ribeiro W.L.C., Macedo I.T.F., Santos J.M.L., Oliveira E.F., Camurça-Vasconcelos A.L.F., Paula H.C.B. \& Bevilaqua C.M.L. 2013. Activity of chitosan-encapsulated Eucalyptus staigeriana essential oil on Haemonchus contortus. Exp. Parasitol. 135:24-29.

Roberts F.H.S. \& O’Sullivan J.P. 1950. Methods for egg counts and larval cultures for strongylesinfsting the gastrointestinal tract of cattle. Aust. J. Agric. Res. 1:99-102.

Rocha F.A.G. \& Dantas L.I.S. 2009. Atividade antimicrobiana in vitro do látex do aveloz (Euphorbia tirucalli L.), pinhão bravo (Jatropha mollissima L.) e pinhão roxo (Jatropha gossypiifolia L.) sobre microrganismos patogênicos. Holos 4:3-11.

Sabandar C.W., Ahmat N., Jaafar F.M. \& Sahidin I. 2013. Medicinal property, phytochemistry and pharmacology of several Jatropha species (Euphorbiaceae). Rev. Phytochem. 85:7-29.

Santos F.C.C., Vogel F.S.F. \& Monteiro S.G. 2012. Extrato aquoso de alho (Allium sativum) sobre nematóides gastrintestinais de ovinos. Revta Bras. Agroecol. 7:139-144.

Santos J.M.L., Monteiro J.P., Ribeiro W.L.C., Macedo I.T.F., Camurça-Vasconcelos A.L.F., Vieira L.S. \& Bevilaqua C.M.L. 2014. Identification and quantification of benzimidazole resistance polymorphisms in Haemonchus contortus isolated in Northeastern Brazil. Vet. Parasitol. 199:160-164.

Sousa R.G., Falcão H.S., Barbosa Filho J.M., Melo Diniz M.F.F. \& Batista L.M. 2013. Atividade anti-helmíntica de plantas nativas do continente americano: uma revisão. Revta Bras. Plant. Med. 15:287-292.

Ueno H. \& Gonçalves P.C. 1998. Manual para diagnóstico das helmintoses de ruminantes. $4^{\text {th }}$ ed. Japan International Cooperation Agency (JICA), Tokyo, Japan. 143p.

Urquhart G.M., Armour J., Duncan J.L., Dunn A.M. \& Jennings F.W. 1998. Parasitologia Veterinária. $2^{\underline{a}}$ ed. Guanabara Koogan, Rio de Janeiro. 273p.

Vieira V.D., Feitosa T.F., Vilela V.L.R., Azevedo S.S., Almeida Neto J.L., Morais D.F., Ribeiro A.R.C. \& Athayde A.C.R. 2014. Prevalence and risk factors associated with goat gastrointestinal helminthiasis in the Sertão region of Paraíba State, Brazil. Trop. Anim. Health Prod. 46:355-361. 